\title{
Acción psicofarmacológica y analgésica del antidepresivo inhibidor selectivo de la recaptación de serotonina fluoxetina en ratón hembra
}

\author{
Lorena Cobo-Realpe ${ }^{1}$, Carlos A. Collazos ${ }^{2}$, Hermes E. Castellanos ${ }^{2}$ y Ketty Herrera-Mendoza ${ }^{3}$ \\ (1) Grupo Biopsiquismo y Sociedad, Universidad Manuela Beltrán, Bogotá D.C, Colombia. \\ (2) Grupo de Ciencias Básicas y Laboratorios, Universidad Manuela Beltrán, Bogotá D.C. Colombia. \\ (e-mail: Iorena.cobor@gmail.com; hsajca@gmail.com; cacollazos@gmail.com) \\ (3) Grupo de Investigación Cultura, Educación y Sociedad. Programa de psicología, Universidad de la Costa CUC, \\ Barranquilla, Colombia. (e-mail: kherrera10@cuc.edu.co)
}

Recibido Oct. 2, 2019; Aceptado Dic. 3, 2019; Versión final Ene. 27, 2020, Publicado Jun. 2020

\begin{abstract}
Resumen
El objetivo de este estudio es dar a conocer el rango de potencia relacionado con la dosificación del ISRS (inhibición selectiva de la recaptación de serotonina) fluoxetina y su mecanismo de acción como agente capaz de inducir efecto analgésico y ansiolítico en ratón hembra cepa CD-1 (25-39 kg). Los datos obtenidos mostraron que las dosis antidepresivas $16,32,64 \mathrm{mg} / \mathrm{kg}$ (i.p.) presentan efecto analgésico y una respuesta ansiolítica, sin resultado significativo en el modelo de memoria espacial, pero con una disminución en la actividad realizada en la memoria de trabajo para la dosis de $64 \mathrm{mg} / \mathrm{kg}$ (i.p.). Esto evidencia que fluoxetina puede orientar mayor eficacia a dosis con efecto antidepresivo e indica un efecto analgésico y ansiolítico sin producir cambio en la actividad motora del ratón. La dosis máxima investigada provocó variación en su actividad motora lo cual permite concluir que ISRS "fluoxetina" tiene un posible efecto sedativo.
\end{abstract}

Palabras clave: inhibidor selectivo; recapatacion de serotonina; fluoxetina; analgesia; antidepresivo

\section{Psychopharmacological and analgesic action of selective serotonin reuptake inhibitor antidepressants fluoxetine in female mouse}

\begin{abstract}
The main objective of this paper is to establish the potency range and the action mechanism of selective serotonin reuptake inhibition (SSRI) fluoxetine on female CD-1 mice $(25-39 \mathrm{~kg})$. Our results indicated that fluoxetine's antidepressant doses of $16 \mathrm{mg} / \mathrm{kg}$ (i.p.), $32 \mathrm{mg} / \mathrm{kg}$ (i.p.), and $64 \mathrm{mg} / \mathrm{kg}$ (i.p.) had an analgesic and anxiolytic effect without significant results on the spatial memory test. Reduced activity on memory work on specific activities was only observed with a dose of $64 \mathrm{mg} / \mathrm{kg}$ (i.p.). This is evidence to suggest that fluoxetine can be more effective with doses that showed an antidepressant effect. It also indicates that fluoxetine can have an analgesic and anxiolytic effect without reducing motor activity on mice. The maximum dose tested on this study showed variations on motor activity of mice suggesting that SSRI fluoxetine can have sedative effects.
\end{abstract}




\section{INTRODUCCIÓN}

El dolor es una manifestación clínica frecuente cuyo tratamiento está lejos de ser óptimo, aunque en los últimos años se han producido avances muy significativos. A esto hay que sumar el hecho de que el dolor suele acompañarse de otras enfermedades comórbidas entre las que se destacan la: depresión, ansiedad, deterioro cognitivo y alteraciones del sueño (Barceló-Martínez, et al. 2018). La mayoría de los fármacos eficaces en el tratamiento de la depresión actúan sobre la neurotransmisión monoaminérgica mediante la inhibición de la recaptación de sus neurotransmisores específicos, dándoles el nombre genérico de "antidepresivos". Las principales dianas para la acción de los antidepresivos son el transportador de serotonina (TSER), el transportador de noradrenalina (TNA) y el transportador de dopamina (TDO) ubicados en la membrana plasmática neuronal. Estos transportadores de monoaminas (TMA) son clave en la regulación de la concentración de neurotransmisores en el espacio sináptico, demostrando que esta neurotransmisión influye de manera importante en aquellos circuitos cerebrales que regulan el estado del ánimo, el estrés, motivación y el rendimiento cognitivo. (Haenisch y Bönisch 2011; Hamon y Blier 2013; Allegri, et al.2019; Delgado-Moreno, et al. 2019) Para el cual se realizan pruebas preclínicas con la intención de afianzar la respuesta de estos antidepresivos en situaciones similares de ansiedad, analgesia, memoria de trabajo y memoria espacial.

En particular, el neurotransmisor de serotonina tiene como fin modular las funciones integradas del sistema nervioso central (S.N.C) implicadas directamente con el estado de ánimo, ansiedad, alimentación y estrés (Delgado-Moreno et al., 2019). Se encuentra localizada en las neuronas de la protuberancia del rafe y el tronco del encéfalo superior, que se proyectan al encéfalo anterior, además, coexisten extensas proyecciones desde la amígdala, el núcleo accumbens, el fascículo estriado anterior, tálamo e hipotálamo. Siendo importante en la neuropsicofarmacología debido a que muchos de los antipsicóticos usados para el tratamiento de la depresión actúan sobre las vías serotoninérgicas. Aunque, entre los antidepresivos altamente selectivos para los trasportadores de serotonina (TSER) están citalopram, escitalopram, fluvoxamina, paroxetina y sertralina.(Hache et al.. 2011; Montoya et al., 2016). A través de los años se incorporaron al mercado otros antidepresivos cuyo mecanismo de acción es inhibir de manera selectiva la recaptación de serotonina (ISRS) como es el caso de fluoxetina (FLX) en 1987, convirtiéndose en el tratamiento no solo para trastornos depresivos sino también actuando de manera eficaz en trastornos de ansiedad, obsesivo-compulsivo, pánico y fobias sino también como analgésico (Montoya et al., 2016; Oliver 2015).

Las pruebas realizadas en este articulo muestran como el fármaco trae una respuesta ansiolítica para modelos experimentales de ansiedad tal es el test de las canicas para las dosis altas, al igual que para los modelos de dolor donde se evidencio un efecto analgésico para modelos de dolor agudo. Siendo fluoxetina el primer antidepresivo en ser aprobado en EE.UU, el cual representó un avance importante en psicofarmacología y ha sido el elemento catalizador de una parte importante de la investigación básica y clínica. A pesar de que los ISRS como grupo farmacológico presentan diversas características comunes, algunos de estos compuestos individuales también muestran características específicas. (Schatzberg y Nemeroff 2016). La mayor parte de 5-HT liberada es recaptada e introducida en la neurona mediante la acción de un transportador. Este sistema de transporte constituye el principal mecanismo de regulación de la concentración de 5-HT intersticial y por tanto de su acceso a los receptores específicos y de la actividad de las neuronas serotoninérgicas. (Ramamoorthy et al., 2011). En los ultimos tiempos se ha hecho muy frecuente, patologias como la depresion mayor y los trastornos de ansiedad a menudo concomitantes (Abello-Luque et al., 2017), implicando la reduccion en la actividad monoaminergica especificamente del sistema serotoninergico. (Vahid-Ansari et al., 2017).

La curva dosis-efecto para cantidades cercanas a los $20 \mathrm{mg} / \mathrm{Kg}$ obtenidas en este escrito, muestran un comportamiento similar a los reportados por Gamil y Nazari los cuales confirman la actividad antidepresiva en modelos predictivos de depresion como el test de natacion forzada en ratas y ratón. Ademas, en el test de suspensión de la cola se observó una disminución en la movilidad con administración oral de fluoxetina de 20 $\mathrm{mg} / \mathrm{Kg}$, lo que reafirma el contenido de monoaminas serotoninergicas en el cerebro cuando el animal realiza el modelo predictivo de depresión. (Gamil et al. 2016;Nazari et al. 2016)

\section{MODELOS EXPERIMENTALES}

Se realizó la evaluación de la actividad motora basada en la valoración de la actividad motora espontánea S.M.A.R.T. ("Spontaneus Motor Activity, Recording and Tracking"), con el fin de conocer la capacidad de movimiento que tenían los animales en las condiciones determinadas por el fármaco investigado, analizando el campo de movimiento de cada animal y convirtiendo en datos numéricos (unidades arbitrarias) la distancia recorrida del animal durante el tiempo previamente estipulado. A continuación se relacionan los modelos experimentales usados y el test asociado a cada prueba. 
Modelos predictivos de actividad antidepresiva: Se utilizo la prueba de natación forzada (FST); de acuerdo con el procedimiento estándar de este modelo, los animales fueron depositados individualmente dentro del recipiente con agua durante 6 min. Sometiendo al animal a una situación aversiva inescapable, contabilizando el tiempo en segundos que el animal se mantuvo inmóvil durante los últimos 4 min del test.(Berrocoso y Micó 2009; Castagné et al., 2011). Para el test de suspensión por la cola (TST), el animal es sometido a una situación inescapable, en este caso el parámetro indicativo de la actividad antidepresiva es el tiempo en segundos (s), que el animal permanece totalmente quieto. La prueba tiene una duración de 6 min. (Castagné et al., 2011).

Modelos de dolor agudo: Se utilizó un estímulo nociceptivo térmico al calor a través de una placa calefactora a temperatura constante de $55 \pm 0.2 \cong \mathrm{C}$. La respuesta refleja la retirada, el lameteo o sacudida de las patas del ratón. En esta prueba el parámetro de medida es el tiempo en segundos de latencia al dolor desde que el animal es colocado en la placa hasta la primera respuesta de alguna de las patas 2traseras. Los resultados fueron analizados y expresados como el porcentaje del tiempo de latencia. (Woolge y MacDonald 1944). Asi mismo, se aplicó el test de administración de ácido acético como respuesta de dolor visceral, que consiste en administrar al animal una solución de ácido acético (C2H4O2 Sigma-Aldrich) diluido en agua destilada al 0.8\% por vía intraperitoneal (i.p.), provocando la constricción tónico - clonicas de sus patas traseras. El criterio de dolor que se tuvo en cuenta fue el número de contorsiones que realizo durante $2 \mathrm{~min}$, tras una latencia de 6 min después de la administración del agente nocivo. (Collier et al.,1968).

Modelos de ansiedad: Se realizó el test de las canicas, el criterio de evaluación fue el número de canicas ocultas por lo menos en dos tercios de su tamaño, durante los 30 min de duración de la prueba. Se considera un efecto ansiogénico, cuando el número de canicas es mayor y por el contrario cuando el número de canicas ocultas es menor o estas permanecen en la posición inicial se puede decir que muestran un efecto ansiolítico. (Sugimoto et al., 2007). Para el test de la caja clara-oscura se colocó al animal en la zona clara del laberinto permitiéndole una libre exploración de una caja a otra durante $5 \mathrm{~min}$, considerando que los espacios abiertos parecen tener propiedades aversivas que impiden un comportamiento exploratorio (zona clara), el parámetro medido fue el tiempo que el animal permaneció en la zona clara durante los 5 min del test. (Majidi-Zolbanin et al., 2013).

Modelos de memoria: El estudio de memoria espacial se realizó con el laberinto de Morris, aca el animal es introducido nadando desde diferentes puntos de partida situados en el perímetro de la piscina, tratando de localizar una plataforma oculta en el fondo del agua durante $60 \mathrm{~s}$, se usaron señales externas como referencia durante la prueba. El parámetro evaluador fue el tiempo que tardo el ratón en encontrar la isla, normalizando los valores (tiempo de recorrido) de cada grupo respecto al primer entrenamiento (dato basal) (Morris 1981). Para memoria de trabajo se utilizó el laberinto radial de ocho brazos. En este caso, los animales son sometidos a una reducción calórica adaptativa. El parámetro experimental para evaluar los efectos del tratamiento fue el porcentaje de precisión de la realización correcta de la tarea. Este valor se calcula partiendo de la fórmula: \% de rendimiento correcto $=$ (número aciertos $/ 8+$ número errores $) \times 100$, normalizando los valores $(\%$ de rendimiento correcto) de cada grupo respecto al primer entrenamiento (dato basal). (Olton 1087).

\section{MATERIALES Y MÉTODOS}

Se utilizaron ratones hembras de la cepa CD-1 (25-30g) suministradas por el servicio de Experimentación y Producción Animal (SEPA) de la Universidad de Cádiz, en condiciones ambientales constantes a una temperatura $21 \pm 1 \% \mathrm{C}$, humedad relativa de $55 \pm 10 \%$, un ciclo continuo de luz-oscuridad de $12 \mathrm{~h}$, con comida y bebida "ad libitum", mediante una dieta comercial estándar. Todos los procedimientos se realizaron siguiendo las recomendaciones que la Federation for Laboratory Animal Science Association (FELASA), establecen en la Unión Europea (directiva 86/609) y en la ley española, (Real Decreto 1201/2005) y aprobados por el Comité de Ética para la Experimentación Animal de la Universidad de Cádiz. Cada grupo experimental estuvo constituido por 10 animales por dosis, el volumen de administración fué de $10 \mathrm{ml} / \mathrm{kg}$, disueltos en solución salina $(\mathrm{NaCl}$ 0,9\%) según indican los resultados en las pruebas de disolución del antidepresivo inhibidor selectivo de la recaptación de serotonina fluoxetina FLX, (Lilly); cuyas dosis empleadas fueron: 4; 8; $16 ; 32 ; 64 \mathrm{mg} / \mathrm{kg}$.

\section{RESULTADOS Y ANÁLISIS}

Los resultados obtenidos se expresan como la media del parámetro referenciado en cada experimento \pm error estándar de la media (E.E.M.). En algunos de los casos y sólo para su representación gráfica, se expresaron los datos como porcentaje respecto al control, equivalente al 100\%. En los modelos de memoria los datos numéricos fueron normalizados respecto al primer día de entrenamiento. El análisis estadístico se realizó con la ayuda del programa estadístico GraphPad Prism (versión 5.00, GraphPad Software). 
Para evaluar la actividad motora se realizó un análisis de varianza (ANOVA) de dos vías de medidas repetidas y posteriormente el test de Bonferroni. Para la evaluación de más de dos grupos categorizados como una vía, se hizo un ANOVA de una vía o de una vía de medidas repetidas, seguido del test de Dunnett. Finalmente, para las comparaciones entre dos grupos, se realizó una $t$ de Student con un grado de significancia $p<0.05$ (Límite de confianza mayor del 95\%).

\section{Fluoxetina sobre la actividad motora.}

Se detallan los resultados de la acción farmacológica en la administración intraperitoneal del antidepresivo inhibidor selectivo de la recaptación de serotonina fluoxetina en ratón hembra obtenidos para la actividad motora y los modelos predictivos de depresión, dolor, ansiedad y memoria. Para estas pruebas se tuvieron en cuenta dosis efectivas en los modelos predictivos de depresión.

Para la actividad motora del antidepresivo ISRS fluoxetina, el ANOVA de dos vías de medidas repetidas reveló un efecto significativo para el factor tratamiento $\left(F_{(5,216)}=66,46 ; p<0,001\right)$, pero no para el factor tiempo $\left(F_{(3,216)}=0,83 ; p>0,05\right)$ y su interacción $\left(F_{(15,216)}=1,01 ; p>0,05\right)$. Posteriormente, aplicando el test post-hoc de Bonferroni, se pudo evidenciar diferencias significativas (indicadas por letras), desde los 5 min hasta los 20 min para las dosis de $32 \mathrm{mg} / \mathrm{kg}(\mathrm{bp}<0,01 ; \mathrm{cp}<0,001)$ y $64 \mathrm{mg} / \mathrm{kg}(\mathrm{cp}<0,001)$, en el sentido de disminución de la actividad motora. Los valores obtenidos para $n=10$ animales por grupo, se relacionan en la tabla 1 y su efecto se indica en la figura 1.

Tabla 1: Valores de Media \pm E.E.M. y porcentajes respecto al control (100\%), obtenidos durante la administración aguda (i.p.) de FLX (actimetría, unidades arbitrarias) 30 min después de la administración y durante 20 min.

\begin{tabular}{|c|c|c|c|c|c|c|c|c|c|c|c|c|c|c|c|c|c|c|}
\hline & \multicolumn{18}{|c|}{ Media \pm E.E.M. } \\
\hline & \multicolumn{3}{|c|}{ Control } & \multicolumn{3}{|c|}{$\mathrm{FLX} 4 \mathrm{mg} / \mathrm{kg}$} & \multicolumn{3}{|c|}{$\mathrm{FLX} 8 \mathrm{mg} / \mathrm{kg}$} & \multicolumn{3}{|c|}{ FLX $16 \mathrm{mg} / \mathrm{kg}$} & \multicolumn{3}{|c|}{ FLX 32 mg/kg } & \multicolumn{3}{|c|}{$\mathrm{FLX} 64 \mathrm{mg} / \mathrm{kg}$} \\
\hline $\min 5$ & 12,0 & \pm & 0,7 & 12,5 & \pm & 1,2 & 11,7 & \pm & 0,8 & 11,7 & \pm & 0,9 & 7,1 & \pm & $0,9 c$ & 4,8 & \pm & $0,7 \mathrm{c}$ \\
\hline $\min 10$ & 8,9 & \pm & 0,5 & 10,5 & \pm & 1,0 & 9,2 & \pm & 0,6 & 9,04 & \pm & 0,8 & 4,2 & \pm & $0,8 \mathrm{c}$ & 1,9 & \pm & $0,3 \mathrm{c}$ \\
\hline $\min 15$ & 7,8 & \pm & 0,9 & 9,3 & \pm & 1,1 & 8,6 & \pm & 0,6 & 7,75 & \pm & 0,9 & 2,6 & \pm & $0,7 \mathrm{c}$ & 1,2 & \pm & $0,2 \mathrm{c}$ \\
\hline \multirow[t]{3}{*}{$\min 20$} & 7,5 & \pm & 0,7 & 9,0 & \pm & 1,1 & 8,2 & \pm & 0,6 & 5,96 & \pm & 1,0 & 2,4 & \pm & $0,9 \mathrm{c}$ & 0,8 & \pm & $0,2 \mathrm{c}$ \\
\hline & \multicolumn{18}{|c|}{ Porcentaje respecto al control (100\%) } \\
\hline & \multicolumn{3}{|c|}{ Control } & \multicolumn{3}{|c|}{$\mathrm{FLX} 4 \mathrm{mg} / \mathrm{kg}$} & \multicolumn{3}{|c|}{$\mathrm{FLX} 8 \mathrm{mg} / \mathrm{kg}$} & \multicolumn{3}{|c|}{$\mathrm{FLX} 16 \mathrm{mg} / \mathrm{kg}$} & \multicolumn{3}{|c|}{$\mathrm{FLX} 32 \mathrm{mg} / \mathrm{kg}$} & \multicolumn{3}{|c|}{$\mathrm{FLX} 64 \mathrm{mg} / \mathrm{kg}$} \\
\hline $\min 5$ & 100,0 & \pm & 5,9 & 105,1 & \pm & 9,9 & 98,3 & \pm & 6,8 & 97,6 & \pm & 8,2 & 58,9 & \pm & $8,33 \mathrm{~b}$ & 40,0 & \pm & $5,71 \mathrm{c}$ \\
\hline $\min 10$ & 100,0 & \pm & 6,3 & 117,5 & \pm & 11,2 & 103,1 & \pm & 7,4 & 101,2 & \pm & 9,9 & 47,1 & \pm & $9,98 \mathrm{c}$ & 20,7 & \pm & $3,80 \mathrm{c}$ \\
\hline $\min 15$ & 100,0 & \pm & 11,9 & 119,5 & \pm & 13,7 & 110,5 & \pm & 8,2 & 99,3 & \pm & 12,0 & 32,6 & \pm & $9,05 \mathrm{c}$ & 15,7 & \pm & $2,87 \mathrm{c}$ \\
\hline $\min 20$ & 100,0 & \pm & 9,6 & 120,4 & \pm & 13,9 & 109,1 & \pm & 8,7 & 79,4 & \pm & 13,3 & 31,5 & \pm & $12,53 \mathrm{c}$ & \begin{tabular}{ll|}
10,7 \\
\end{tabular} & \pm & $2,61 \mathrm{c}$ \\
\hline
\end{tabular}

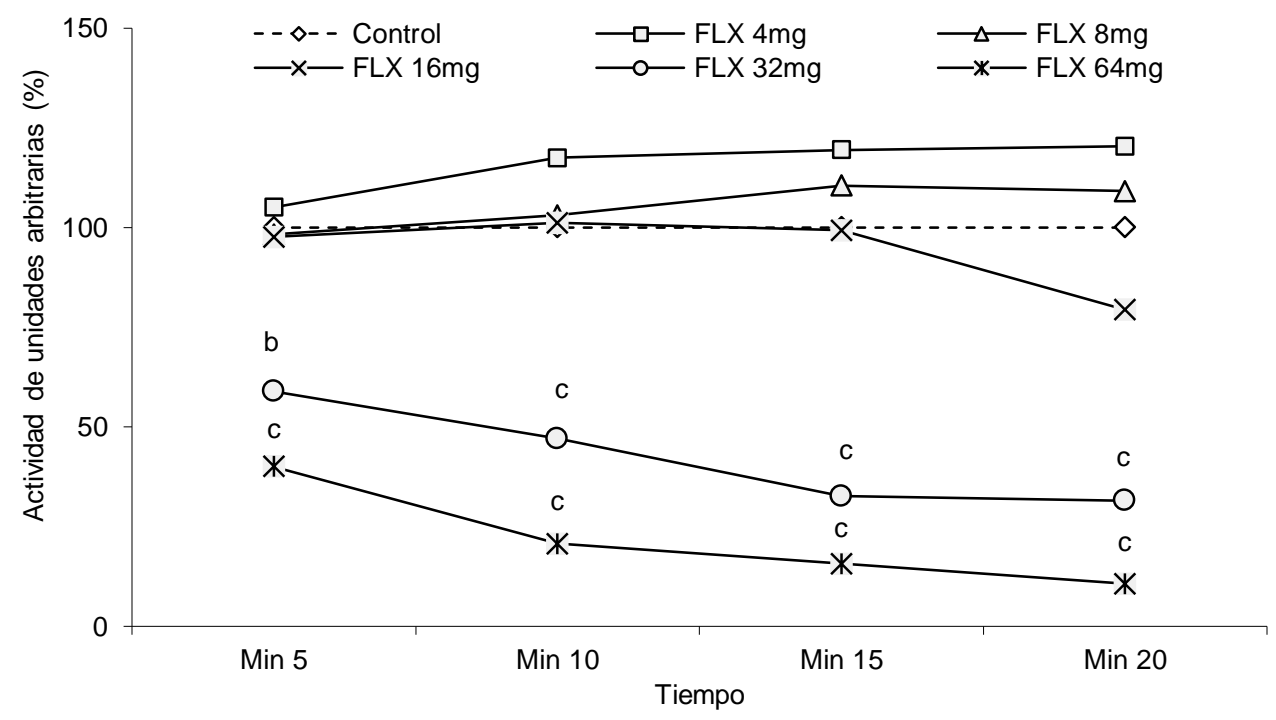

Fig 1: Efecto de la administración aguda (i.p.) de FLX (actimetría, unidades arbitrarias), durante el test post-hoc de Bonferroni. 


\section{Modelos predictivos de depresión}

En el modelo predictivo de actividad antidepresiva sobre la inmovilidad para el test de natación forzada el resultado obtenido con el ANOVA de una vía para el tiempo de inmovilidad, reveló un efecto significativo para la variable tratamiento $\left(F_{(5,47)}=19,73 ; \mathrm{p}<0,001\right)$. Posteriormente, el test de Dunnett mostró diferencias significativas (indicadas por letras) en la disminución del tiempo de inmovilidad para las dosis de $16 \mathrm{mg} / \mathrm{kg}$ $(\mathrm{ap}<0,05) ; 32$ y $64 \mathrm{mg} / \mathrm{kg}(\mathrm{cp}<0,001)$ comparado con el grupo control. Los valores obtenidos para $\mathrm{n}=10$ animales por grupo, se relacionan en la tabla 2 y su efecto se indica en la figura 2.

Tabla 2: Valores de Media \pm E.E.M. y porcentajes respecto al control (100\%), del efecto de FLX sobre la inmovilidad; para los últimos 4 min en el test de la natación forzada en ratón y 30 min después de la administración del fármaco.

\begin{tabular}{|c|c|c|c|c|c|c|}
\cline { 2 - 7 } \multicolumn{1}{c|}{} & \multicolumn{3}{c|}{ Media \pm E.E.M. } & \multicolumn{3}{c|}{ Porcentaje respecto al control (100\%) } \\
\hline Control & 185,7 & \pm & 5,6 & 100,0 & \pm & 3,1 \\
\hline FLX 4 mg/kg & 158,2 & \pm & 12,6 & 85,2 & \pm & 6,7 \\
\hline FLX $8 \mathrm{mg} / \mathrm{kg}$ & 187,6 & \pm & 7,5 & 101,0 & \pm & 4,1 \\
\hline FLX $16 \mathrm{mg} / \mathrm{kg}$ & 125,9 & \pm & $25,4 \mathrm{a}$ & 67,8 & \pm & $13,7 \mathrm{a}$ \\
\hline FLX $32 \mathrm{mg} / \mathrm{kg}$ & 50,44 & \pm & $15,9 \mathrm{c}$ & 27,1 & \pm & $8,6 \mathrm{c}$ \\
\hline FLX $64 \mathrm{mg} / \mathrm{kg}$ & 26,89 & \pm & $13,7 \mathrm{c}$ & 16,2 & \pm & $8,1 \mathrm{c}$ \\
\hline
\end{tabular}

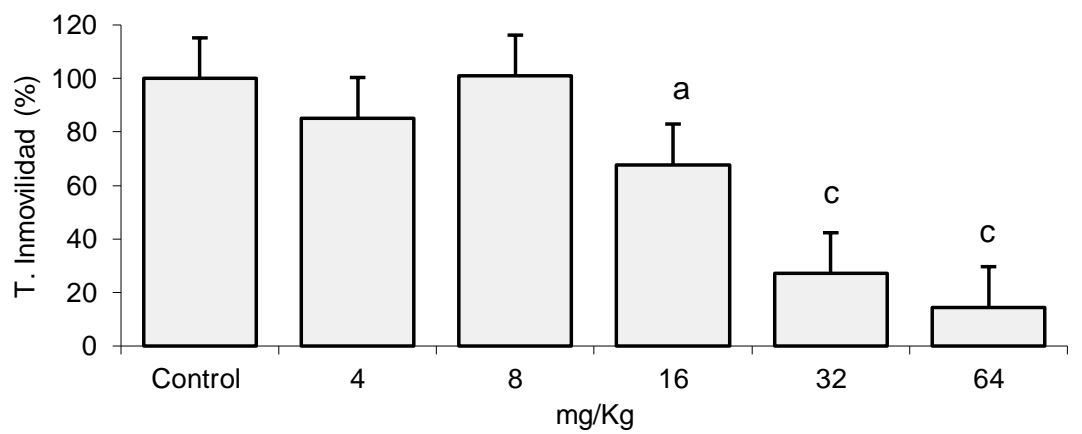

Fig 2: Efecto de FLX sobre la inmovilidad durante el test de la natación forzada en ratón.

Inmovilidad en el test de suspensión por la cola

El resultado estadístico en el ANOVA de una vía para el tiempo de inmovilidad reveló un efecto significativo para el factor tratamiento $\left(F_{(5,53)}=5,934 ; p<0,001\right)$. Posteriormente, el test post-hoc de Dunnett, mostró una disminución significativa (indicadas por letras) en el tiempo de inmovilidad para las dosis de 32 y $64 \mathrm{mg} / \mathrm{kg}$ (c $p<0,001$ ) comparado con el grupo control. Los valores obtenidos para $n=10$ animales por grupo, se relacionan en la tabla 3 y su efecto se indica en la figura 3.

Tabla 3: Valores de Media \pm E.E.M. y porcentajes respecto al control (100\%), del efecto de FLX sobre la inmovilidad durante el TST, 30 min después de la administración del fármaco.

\begin{tabular}{|c|c|c|c|c|c|c|}
\cline { 2 - 8 } \multicolumn{1}{c|}{} & \multicolumn{3}{c|}{ Media \pm E.E.M. } & \multicolumn{3}{c|}{ Porcentaje respecto al control (100\%) } \\
\hline Control & 119,9 & \pm & 18,5 & 100,0 & \pm & 15,5 \\
\hline FLX 4 mg/kg & 93,0 & \pm & 13,9 & 77,5 & \pm & 11,6 \\
\hline FLX $8 \mathrm{mg} / \mathrm{kg}$ & 104,0 & \pm & 19,9 & 86,7 & \pm & 16,6 \\
\hline FLX $16 \mathrm{mg} / \mathrm{kg}$ & 72,2 & \pm & 14,3 & 60,2 & \pm & 11,9 \\
\hline FLX $32 \mathrm{mg} / \mathrm{kg}$ & 36,7 & \pm & $10,5 \mathrm{c}$ & 30,6 & \pm & $8,87 \mathrm{c}$ \\
\hline FLX 64 mg/kg & 34,7 & \pm & $5,2 \mathrm{c}$ & 28,9 & \pm & $4,30 \mathrm{c}$ \\
\hline
\end{tabular}




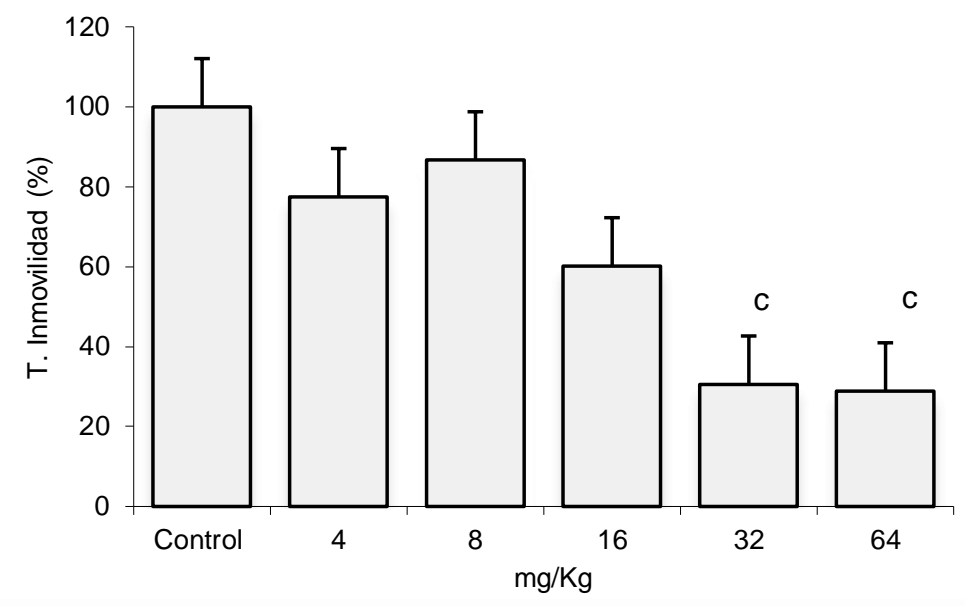

Fig 3: Efecto de FLX sobre la inmovilidad durante el TST.

Modelos de dolor agudo

El ANOVA de una vía para el tiempo de latencia al dolor reveló un efecto significativo para la variable tratamiento $\left(F_{(5,54)}=6.165 ; p<0,001\right)$. Posteriormente, el test de Dunnett mostró en el tiempo de latencia, diferencias significativas (indicadas por letras) para las dosis de $32 \mathrm{mg} / \mathrm{kg}(\mathrm{bp}<0,01)$ y $64 \mathrm{mg} / \mathrm{kg}(\mathrm{cp}<0,001)$ comparado con el grupo control. Los valores obtenidos para $n=10$ animales por grupo, se relacionan en la tabla 4 y su efecto se indica en la figura 4.

Tabla 4: Valores de Media \pm E.E.M. y porcentajes respecto al control (100\%), del efecto de la administración aguda (i.p.) de FLX sobre el tiempo de latencia al dolor, 30 min después de la administración del fármaco.

\begin{tabular}{|c|c|c|r|c|l|l|}
\cline { 2 - 7 } \multicolumn{1}{c|}{} & \multicolumn{3}{c|}{ Media \pm E.E.M. } & \multicolumn{3}{c|}{ Porcentaje respecto al control (100\%) } \\
\hline Control & 6,0 & \pm & 0,6 & 100,0 & \pm & 9,8 \\
\hline FLX 4 mg/kg & 6,6 & \pm & 1,6 & 109,1 & \pm & 25,8 \\
\hline FLX $8 \mathrm{mg} / \mathrm{kg}$ & 8,7 & \pm & 1,9 & 144,2 & \pm & 31,3 \\
\hline FLX $16 \mathrm{mg} / \mathrm{kg}$ & 12,6 & \pm & 2,1 & 209,5 & \pm & 34,3 \\
\hline FLX $32 \mathrm{mg} / \mathrm{kg}$ & 17,9 & \pm & $2,8 \mathrm{~b}$ & 297,8 & \pm & $45,9 \mathrm{~b}$ \\
\hline FLX $64 \mathrm{mg} / \mathrm{kg}$ & 21,2 & \pm & $4,5 \mathrm{c}$ & 353,3 & \pm & $74,7 \mathrm{c}$ \\
\hline
\end{tabular}

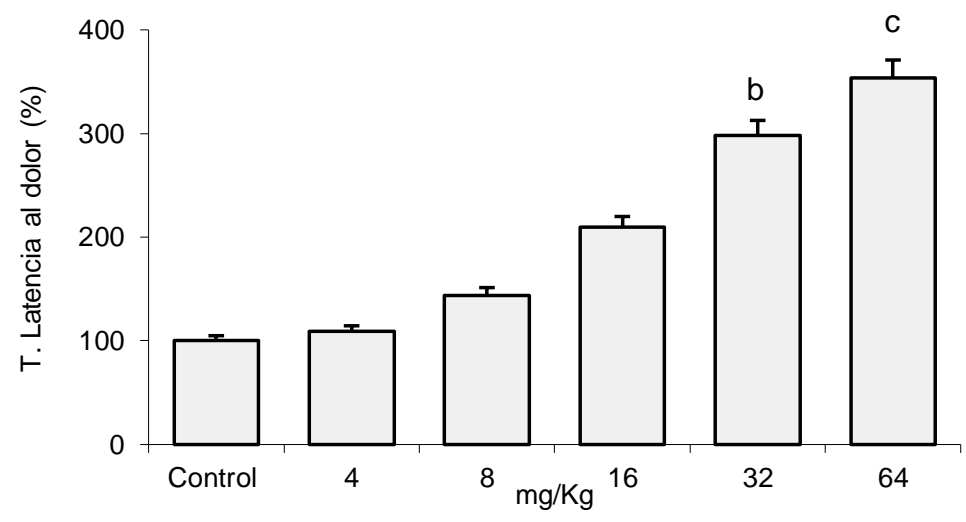

Fig 4: Porcentaje de latencia al dolor durante el test de la placa caliente.

Número de contorsiones en el test del ácido acético

Según el análisis estadístico, el ANOVA de una vía para el número de contorsiones inducidas por la solución de ácido acético reveló un efecto significativo para la variable tratamiento $\left(F_{(5,54)}=14,1 ; p<0,001\right)$. Posteriormente, el test de Dunnett mostró diferencias significativas (indicadas por letras) para las dosis de 16, 32 y $64 \mathrm{mg} / \mathrm{kg}$ comparado con el grupo control $(\mathrm{cp}<0,001)$, en el sentido de disminuir el número de contorsiones inducidas por la solución de ácido acético. Los valores obtenidos para $n=10$ animales por grupo, se relacionan en la tabla 5 y su efecto se indica en la figura 5 . 
Tabla 5: Valores de Media \pm E.E.M. y porcentajes respecto al control (100\%), del efecto de la administración aguda de FLX sobre el número de contorsiones inducidas por la solución de ácido acético en ratón, 30 min después de la administración del fármaco.

\begin{tabular}{|c|c|c|c|c|c|c|}
\cline { 2 - 7 } \multicolumn{1}{c|}{} & \multicolumn{3}{c|}{ Media \pm E.E.M. } & \multicolumn{3}{c|}{ Porcentaje respecto al control (100\%) } \\
\hline Control & 6,7 & \pm & 0,7 & 100,0 & \pm & 9,9 \\
\hline FLX 4 mg/kg & 4,8 & \pm & 0,8 & 71,6 & \pm & 11,5 \\
\hline FLX $8 \mathrm{mg} / \mathrm{kg}$ & 4,3 & \pm & 0,9 & 64,2 & \pm & 13,2 \\
\hline FLX $16 \mathrm{mg} / \mathrm{kg}$ & 1,8 & \pm & $0,7 \mathrm{c}$ & 26,9 & \pm & $10,4 \mathrm{c}$ \\
\hline FLX $32 \mathrm{mg} / \mathrm{kg}$ & 0,9 & \pm & $0,6 \mathrm{c}$ & 13,4 & \pm & $9,02 \mathrm{c}$ \\
\hline FLX $64 \mathrm{mg} / \mathrm{kg}$ & 0,3 & \pm & $0,1 \mathrm{c}$ & 4,5 & \pm & $2,3 \mathrm{c}$ \\
\hline
\end{tabular}

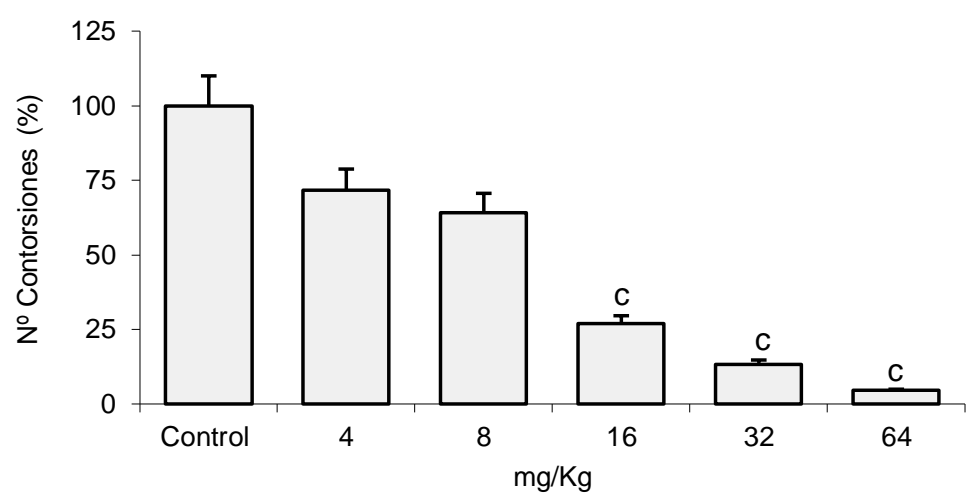

Fig 5: Efecto de fluoxetina en el modelo de ácido acético, durante el test de Dunnett.

\section{Modelos de ansiedad}

El resultado estadístico de la acción farmacológica de FLX en el ANOVA de una vía para el número de canicas ocultas, reveló un efecto significativo para la variable tratamiento $\left(F_{(3,36)}=24,10 ; p<0,001\right)$. El test post-hoc demostró diferencias significativas (indicadas por letras) para las dosis de 16, 32 y 64mg/kg en comparación con el grupo control ( $c p<0,001$; Test de Dunnett). Los valores obtenidos para $n=10$ animales por grupo, se relacionan en la tabla 6 y su efecto se indica en la figura 6.

Tabla 6: Valores de Media \pm E.E.M. y porcentajes respecto al control (100\%), del efecto de la administración aguda (i.p.) de FLX sobre el número de canicas ocultas, 30 min después de la administración del fármaco.

\begin{tabular}{|c|c|c|c|c|c|c|}
\cline { 2 - 7 } \multicolumn{1}{c|}{} & \multicolumn{3}{c|}{ Media \pm E.E.M. } & \multicolumn{3}{c|}{ Porcentaje respecto al control (100\%) } \\
\hline Control & 10,6 & \pm & 0,4 & 100,0 & \pm & 4,0 \\
\hline FLX $16 \mathrm{mg} / \mathrm{kg}$ & 5,20 & \pm & $1,5 \mathrm{c}$ & 49,1 & \pm & $14,3 \mathrm{c}$ \\
\hline FLX $32 \mathrm{mg} / \mathrm{kg}$ & 1,70 & \pm & $0,8 \mathrm{c}$ & 16,0 & \pm & $8,2 \mathrm{c}$ \\
\hline FLX $64 \mathrm{mg} / \mathrm{kg}$ & 0,6 & \pm & $0,3 \mathrm{c}$ & 5,7 & \pm & $3,2 \mathrm{c}$ \\
\hline
\end{tabular}

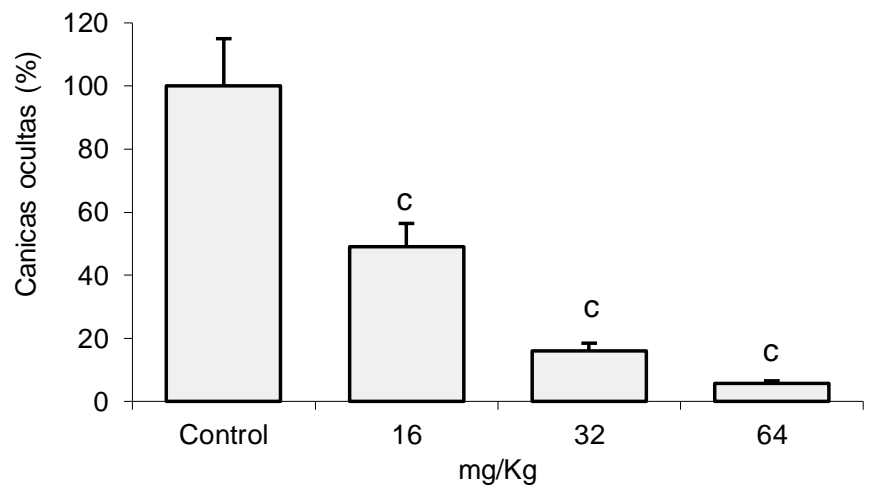

Fig 6: Efecto de la administración aguda (i.p.) de FLX, durante el test de las canicas. 


\section{Tiempo de permanencia en test claro oscuro}

El resultado obtenido en el ANOVA de una vía para el tiempo de permanencia en la zona clara del test, no 2 mostró ningún efecto significativo para el tratamiento $\left(F_{(3,36)}=2,431 ; p>0,05\right)$. Posteriormente en el test posthoc no hubo diferencias significativas para ninguna de las dosis, respecto a el grupo control $(p>0,05$; Test de Dunnett). Los valores obtenidos para $\mathrm{n}=10$ animales por grupo, se relacionan en la tabla 7 y se representan en la figura 7.

Tabla 7: Valores de Media \pm E.E.M. y porcentajes respecto al control (100\%), de administración aguda de FLX sobre el tiempo de permanencia en la zona clara de la caja clara-oscura en ratón 30 min después de la administración del fármaco.

\begin{tabular}{|c|c|c|c|c|c|c|}
\cline { 2 - 7 } \multicolumn{1}{c|}{} & \multicolumn{3}{c|}{ Media \pm E.E.M. } & \multicolumn{3}{c|}{ Porcentaje respecto al control (100\%) } \\
\hline Control & 101,0 & \pm & 12,7 & 100,0 & \pm & 12,6 \\
\hline FLX 16 mg/kg & 96,4 & \pm & 5,5 & 95,2 & \pm & 5,4 \\
\hline FLX 32 mg/kg & 119,0 & \pm & 21,7 & 117,7 & \pm & 21,4 \\
\hline FLX 64 mg/kg & 163,0 & \pm & 29,3 & 161,2 & \pm & 28,9 \\
\hline
\end{tabular}

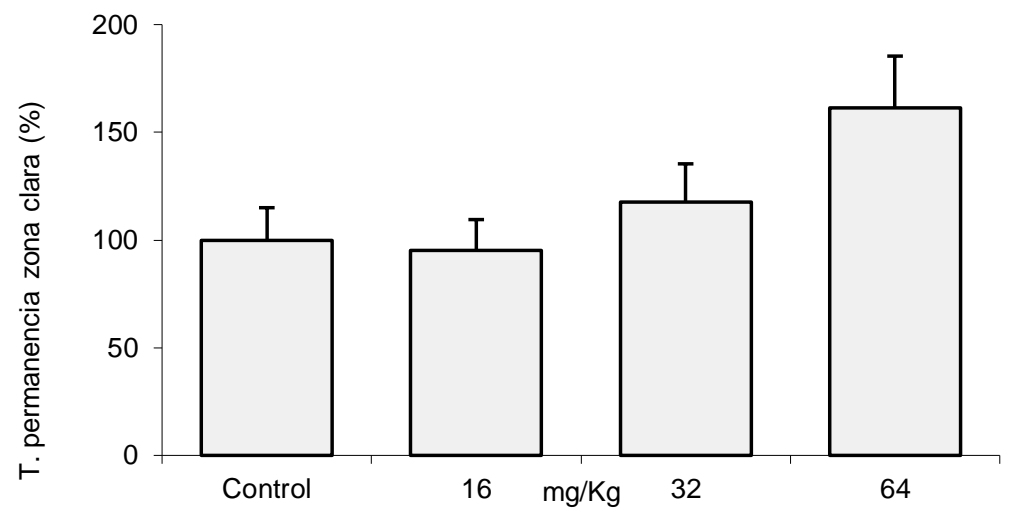

Fig 7: Tiempo de permanencia en la zona clara de la caja clara-oscura en ratón 30 min después de la administración del fármaco, durante el test de caja clara-oscura.

\section{Modelo de memoria espacial}

El resultado obtenido en el ANOVA de una vía para el tiempo normalizado de cada grupo mostró un efecto significativo para el factor tratamiento $\left(F_{(3,36)}=3,693 ; p<0,05\right)$. Posteriormente, un test post-hoc no ofreció diferencias significativas en el porcentaje normalizado de cada grupo para ninguna de las dosis, comparadas con el grupo control ( $p>0,05$; test de Dunnett). Los valores obtenidos para $n=10$ animales por grupo, se relacionan en la tabla 8 y su efecto se indica en la figura 8.

Tabla 8: Valores de Media \pm E.E.M. del efecto de la administración aguda (i.p.) de FLX en el laberinto de Morris en ratón, durante 1 minuto, 30 min después de la administración del fármaco.

\begin{tabular}{|c|c|c|c|}
\cline { 2 - 4 } \multicolumn{1}{c|}{} & \multicolumn{3}{c|}{ Media \pm E. E.M. } \\
\hline Control & 107,6 & \pm & 19,3 \\
\hline FLX 16 mg/kg & 50,7 & \pm & 13,4 \\
\hline FLX 32 mg/kg & 69,5 & \pm & 14,6 \\
\hline FLX 64 mg/kg & 144,4 & \pm & 33,2 \\
\hline
\end{tabular}

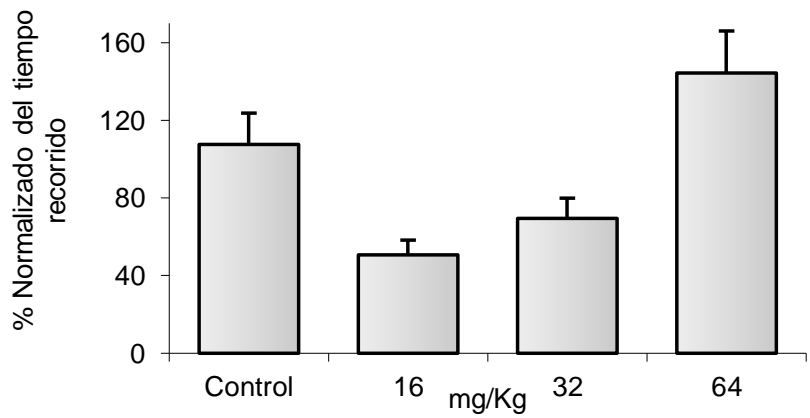

Fig 8: Efecto de la administración ip de FLX en el recorrido, dentro del laberinto de Morris. 


\section{Modelo de memoria de trabajo}

EI ANOVA de una vía para el porcentaje normalizado del rendimiento correcto en el laberinto de ocho brazos, mostró un efecto significativo para el tratamiento $\left(F_{(3,36)}=3,260\right.$; a $\left.p<0,05\right)$. Posteriormente, el test post-hoc ofreció diferencia significativa (indicadas por letras) en el tratamiento para la dosis de $64 \mathrm{mg} / \mathrm{kg}$, en relación con el grupo control (a $p<0,05$; test de Dunnett). Los valores obtenidos para $n=10$ animales por grupo, se relacionan en la tabla 9 y su rendimiento se indica en la figura 8.

Tabla 9: Valores de Media \pm E.E.M. del efecto de la administración aguda (i.p.) de FLX sobre el porcentaje normalizado de rendimiento en el laberinto de ocho brazos en ratón, 30 min después de la administración del fármaco.

\begin{tabular}{|c|c|c|l|}
\cline { 2 - 4 } \multicolumn{1}{c|}{} & \multicolumn{3}{c|}{ Media \pm E.E.M. } \\
\hline Control & 100,9 & \pm & 13,6 \\
\hline FLX 16 mg/kg & 105,5 & \pm & 11,6 \\
\hline FLX 32 mg/kg & 84,2 & \pm & 15,4 \\
\hline FLX 64 mg/kg & 52,2 & \pm & $12,3 \mathrm{a}$ \\
\hline
\end{tabular}

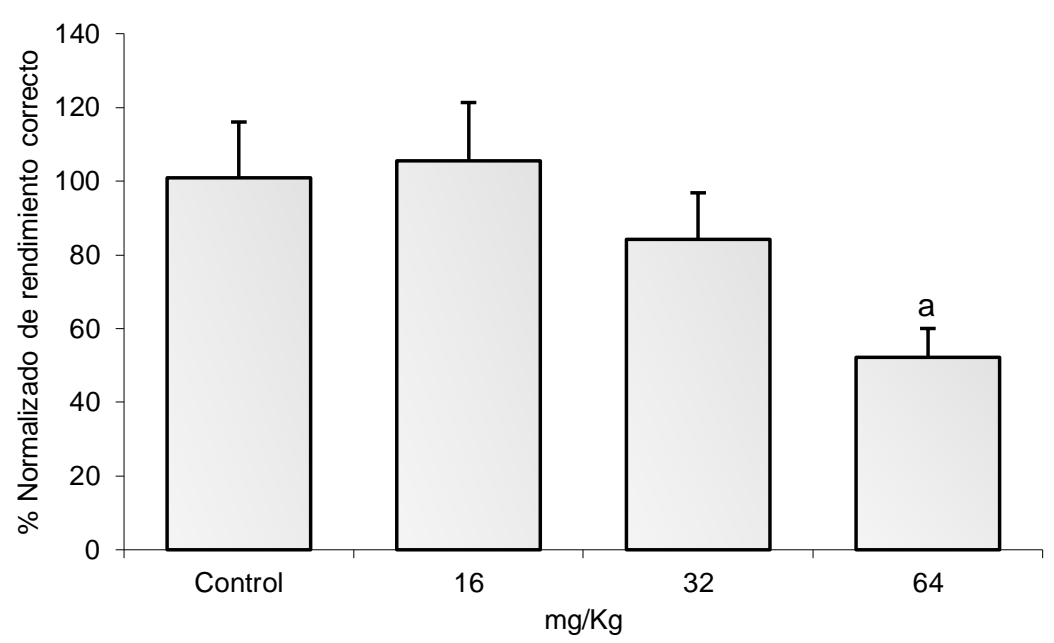

Fig 9: Porcentaje normalizado del rendimiento en el laberinto de 8 brazos.

\section{DISCUSIÓN}

Diferentes estudios preclínicos han demostrado que los cambios en la actividad motora podrían ser influenciados por los efectos del tratamiento inhibitorio sobre esta función, este argumento soporta los resultados obtenidos en el estudio donde las dosis altas de fluoxetina las cuales derivaron en una reducción significativa del comportamiento exploratorio a lo largo del tiempo, estos resultados se confirma con aquellos obtenidos en el 2009 por Berrocoso y cols donde la movilidad del animal disminuyo de manera significativa a la dosis de $40 \mathrm{mg} / \mathrm{kg}$ de fluoxetina. (Berrocoso y Micó 2009; Umathe y Jain 2011). Para los modelos predictivos de depresión como el test de natación forzada y el test de suspensión de la cola se corrobora el efecto antidepresivo del fármaco ISRS para dosis mayores de $16 \mathrm{mg}$ ya que muestran mayor sensibilidad en el tiempo de inmovilidad tal como lo indica Karim y colaboradores en su estudio del efecto antidepresivo de fluoxetina (Karim et al., 2018), y otros trabajos donde utilizaron una dosis antidepresiva del fármaco como control positivo para sus estudios. ( Souza et al., 2018; Umathe et al., 2011).

Por otra parte, el dolor al ser una patología de difícil tratamiento ya que aún falta mucho por estudiar sobre los mecanismos neurobiológicos subyacentes. Hace que sea necesario profundizar en el conocimiento de las bases moleculares y neurológicas con el fin de obtener terapias para su tratamiento (Barceló-Martínez et al., 2018). La acción farmacológica de los antidepresivos en el tratamiento del dolor es un hecho conocido desde hace más de 50 años, varios estudios así lo han demostrado. (Cobo-Realpe et al., 2012; Micó et al., 2006). Siendo crucial en la validación de un antidepresivo como analgésico, es por esto que en esta investigación se estudió el inhibidor selectivo para la recaptación de serotonina en modelos de dolor térmico y visceral con el propósito de observar los posibles efectos analgésico del tratamiento y la presencia viable de los factores asociados. Estudios que dieron resultados con una tendencia antinociceptiva a dosis bajas siendo efectiva como analgésico a partir de $16 \mathrm{mg} / \mathrm{kg}$, sugiriendo que FLX, a pesar de su efecto colateral sobre el comportamiento exploratorio, igualmente produce un posible efecto analgésico que puede considerarse independiente. La administración aguda de altas dosis de FLX produjo un efecto analgésico en el modelo de la placa caliente, aumentando el tiempo de latencia al estímulo térmico doloroso. 
Del mismo modo, se observó un bloqueo dosis-dependiente de las contorsiones realizadas por el animal en el test del ácido acético a partir de $16 \mathrm{mg} / \mathrm{kg}$. Contrario al resultado obtenido por Hall y col en el 2011 donde se utilizaron sepas Knockout de serotonina por ende la respuesta dosis efecto de fluoxetina para el test de la placa caliente no evidencio efecto analgésico. (Hall et al., 2011). Contrario al resultado obtenido por Schreiber donde la respuesta de nociceptiva de fluoxetina fue débil. (Schreiber y Pick 2006). Teniendo en cuenta las respuestas efectivas en estos modelos mencionados anteriormente, se probaron las dosis que mostraron tanto un efecto antidepresivo como analgésico, para la evaluación de los modelos de ansiedad y de memoria tanto de trabajo como espacial.

\section{CONCLUSIONES}

De acuerdo a los resultado se puede concluir que ISRS "fluoxetina" a dosis máxima depresiva, 1) Indujo variaciones en la activiad motora refiniendo un posible efecto sedativo. 2) Se identifico un efecto analgésico al dolor agudo en modelos de dolor y una respuesta ansiolítica ocultando objetos sin afectar la motricidad del animal. 3) La adminstracion de la dosis máxima afecto en la memoria espacial, evidencio una posible relación con la disminución de la actividad motora.4) La administración de ISRS disminuye de manera significativa el número de canicas ocultas en la dosis intermedia y esta respuesta se mantiene en las dosis para el tratamiento más alto, presentando un efecto ansiolítico. Esto respaldado con los experimentos iniciales donde se evaluó la actividad motora espontanea cuyo resultado presenta actividad para la dosis de $16 \mathrm{mg} / \mathrm{Kg}$, por lo que se tiene la certeza de su respuesta ansiolítica, de acuerdo con los efectos obtenidos en otros estudios donde se redujo el comportamiento impulsivo de los ratones al ocultar las canicas para la dosis de $10 \mathrm{mg} / \mathrm{Kg} ; 20$ y 30 $\mathrm{mg} / \mathrm{Kg}$ de fluoxetina. (Grassi et al., 2016: Umathe et al., 2011). 5) En el test de la caja clara-oscura se observa una tendencia al aumentar la exploración en el lado claro, lo que indica el efecto de luminosidad aversivo no es molesto para las dosis administradas, por lo que se puede argumentar que fluoxetina en las dosis establecida no produce un efecto ansiolítico.

\section{REFERENCIAS}

Abello-Luque, D., Cortes-Peña. O y otros 4 autores. "Escala Multidimensional de Trastornos Afectivos, EMTA: Generación de Indicadores Psicométricos y Escalas Normativas de Uso Clínico". https://doi.org/10.21865/RIDEP43_187 Revista Iberoamericana de Diagnóstico y Evaluación. 43(1): 187-198. (2017).

Allegri, R., Pertierra, L. y otros 10 autores. "A biological classification for Alzheimer's disease - Amyloid, Tau and Neurodegeneration $(A / T / N)$ : results from the Argentine-Alzheimer's Disease Neuroimaging Initiative". https://doi.org/10.1017/S1041610219000085 International Psychogeriatrics. 1-2. (2019).

Barceló-Martinez, E., Gelves-Ospina, M. y otros 5 autores. "Niveles de cortisol sérico y alteraciones neuropsicológicas en pacientes con diagnóstico de Fibromialgia”. Actas Españolas de Psiquiatria. 46(1):1-11. (2018).

Berrocoso, E. y Micó. J. A. "Cooperative Opioid and Serotonergic Mechanisms Generate Superior Antidepressant-like Effects in a Mice Model of Depression." https://doi.org/10.1017/S1461145709000236 International Journal of Neuropsychopharmacology 12: 1033-44. (2009).

Castagné, V. Moser, P. y otros 2 autores. "Rodent Models of Depression: Forced Swim and Tail Suspension Behavioral Despair Tests in Rats and Mice." https://doi.org/10.1002/0471142301.ns0810as55 Current Protocols in Neuroscience (SUPPL.55): 1-14. (2011)

Cobo-Realpe, B.L., y otros 4 autores. "Antidepressant Drugs and Pain." In Effects of Antidepressants, https://doi.org/10.5772/48022 Croatia, 143-62. (2012).

Collier, J, y otros 2 autores. "The Abdominal Constriction Response and its Suppression by Analgesic Drugs in the Mouse." $32 \mathrm{Br}$. J. Pharmac. Chemother. (1968).

Delgado-Moreno, R., Robles-Pérez, J., y otros 2 autores. "Effect of Experience and Psychophysiological Modification by Combat Stress in Soldier's Memory". https://doi.org/10.1007/s10916-019-1261-1 Journal of Medical Systems. 43:150. 28. (2019).

Gamil, N. M. y otros 4 autores. "Modulatory Effect of Cilostazol on Tramadol-Induced Behavioral and Neurochemical Alterations in Rats Challenged across the Forced Swim Despair Test." DOI: 10.1016/j.bjbas.2016.03.002 Beni-Suef University Journal of Basic and Applied Sciences 5(2): 193-201. (2016).

Grassi, G. y otros 6 autores. "Atomoxetine for Hoarding Disorder: A Pre-Clinical and Clinical Investigation." DOI: 10.1016/j.jpsychires.2016.09.012 Journal of Psychiatric Research 83: 240-48. (2016).

Hache, G., Coudore, F y otros 2 autores. "Monoaminergic Antidepressants in the Relief of Pain: Potential Therapeutic Utility of Triple Reuptake Inhibitors (TRIs)." Pharmaceuticals. (2011).

Haenisch, y otros 2 autores.. "Depression and Antidepressants: Insights from Knockout of Dopamine, Serotonin or Noradrenaline Re-Uptake Transporters." https://doi.org/10.1016/j.pharmthera.2010.12.002 Pharmthera.2010.12.002 Pharmacology \& Therapeutics 129 (3): 352-68 (2011). 
Hall, F S y otros 7 autores. "A Greater Role for the Norepinephrine Transporter than the Serotonin Transporter in Murine Nociception.” https://doi.org/10.1016/j.neuroscience.2010.11.057 Neuroscience 175: 315-27. (2011).

Hamon, M., y Pierre B. "Monoamine Neurocircuitry in Depression and Strategies for New Treatments." https://doi.org/10.1016/j.pnpbp.2013.04.009. Progress in Neuro-Psychopharmacology and Biological Psychiatry 45: 5463. (2013)

Karim, N. y otros 4 autores. "Antidepressant Potential of Novel Flavonoids Derivatives from Sweet Violet (Viola Odorata L): Pharmacological, Biochemical and Computational Evidences for Possible Involvement of Serotonergic Mechanism." https://doi.org/10.1016/j.fitote.2018.05.016 Fitoterapia 128: 148-61.(2018).

Majidi-Zolbanin, J. y otros 4 autores. "Adolescent Fluoxetine Treatment Decreases the Effects of Neonatal Immune Activation on Anxiety-like Behavior in Mice." https://doi.org/10.1016/j.bbr.2013.05.003 Behavioural Brain Research 250: 123-32. (2013)

Micó, J. y otros 3 autores "Antidepressants and Pain." https://doi.org/10.1016/j.tips.2006.05.004 Trends in Pharmacological Sciences 27(7): 348-54. (2006).

Montoya, A., Bruins, R y otros 2 autores. "The Noradrenergic Paradox: Implications in the Management of Depression and Anxiety." https://doi.org/10.2147/NDT.S91311 Neuropsychiatric Disease and Treatment 12: 541. (2016).

Morris, R., "Spatial Localization Does Not Require the Presence of Local Cues." https://doi.org/10.1016/00239690(81)90020-5 Learning and Motivation 12(2): 239-60. (1981).

Nazari, S. K. y otros 5 autores. "Possible Involvement of ATP-Sensitive Potassium Channels in the Antidepressant-like Effect of Baclofen in Mouse Forced Swimming Test." https://doi.org/10.1016/j.pharep.2016.07.006 Pharmacological Reports 68(6): 1214-20. (2016)

Oliver, B. "Serotonin: A Never-Ending Story." https://doi.org/10.1016/j.ejphar.2014.10.031 European Journal of Pharmacology 753: 2-18. (2015).

Olton, D. S. "The Radial Arm Maze as a Tool in Behavioral Pharmacology." https://doi.org/10.1016/0031-9384(87)902861 Physiology \& Behavior 40(6): 793-97. (1987)

Ramamoorthy, S., y otros 2 autores.. "Regulation of Monoamine Transporters: Role of Transporter Phosphorylation" https://doi.org/10.1016/j.pharmthera.2010.09.009 Pharmacol and Therapeutics 129(2):220-238 (2011)

Schatzberg, A. F, y Nemeroff, C. B. "Tratado de Psicofarmacología." (January): 1-226. (2006)

Schreiber, S., y Pick C. G.. "From Selective to Highly Selective SSRIs: A Comparison of the Antinociceptive Properties of Fluoxetine, Fluvoxamine, Citalopram and Escitalopram." https://doi.org/10.1016/j.euroneuro.2005.11.013 European Neuropsychopharmacology 16(6): 464-68. (2006).

De Souza, J. M. y otros 6 autores "Zinc Oxide Nanoparticles in Predicted Environmentally Relevant Concentrations Leading to Behavioral Impairments in Male Swiss Mice." https://doi.org/10.1016/j.scitotenv.2017.09.051 Science of The Total Environment 613-614: 653-62. (2018)

Sugimoto, Y. y otros 4 autores. "Effects of the Serotonin and Noradrenaline Reuptake Inhibitor (SNRI) Milnacipran on Marble Burying Behavior in Mice.” https://doi.org/10.1248/bpb.30.2399 Pharm. Bull 30(12): 2399-2401. (2007).

Umathe, S. N. y otros 2 autores. "Involvement of Endocannabinoids in Antidepressant and Anti-Compulsive Effect of Fluoxetine in Mice." https://doi.org/120.1016/j.bbr.2011.04.031 Behavioural Brain Research 223(1): 125-34. (2011)

Vahid-Ansari, F. y otros 9 autores. "Abrogated Freud-1/Cc2d1a Repression of 5-HT1A Autoreceptors Induces FluoxetineResistant Anxiety/Depression-Like Behavior." https://doi.org/10.1523/JNEUROSCI.1668-17.2017 The Journal of Neuroscience 37 (2017) 
